\title{
Influence of vibrational anharmonicity and vacancies on thermodynamic properties of the rare gas crystals
}

\author{
A.I. Karasevskii \\ Institute for Metal Physics of the National Academy of Science of Ukraine \\ 36 Vernadsky Blvd., Kiev 03142, Ukraine \\ E-mail: akaras@imp.kiev.ua \\ W.B. Holzapfel \\ Department Physik, Universität Paderborn, D-33095 Paderborn, Germany
}

\begin{abstract}
A statistical method is used to calculate thermodynamic properties of $\mathrm{Ar}, \mathrm{Kr}$ and $\mathrm{Xe}$ (isobaric and isochoric heat capacity, bulk modulus, thermal expansion coefficient, interatomic distances, Grüneisen parameter), and good agreement with experimental values is observed. It is shown that at high temperature, slightly above the melting point of the rare gas crystals, an instability of the crystalline state occurs. As temperature approaches this instability the isobaric heat capacity and the thermal expansion coefficient show strong increases similar to the experimentally observed anomalies.
\end{abstract}

PACS: 63.70.+h, 64.30.+t, 05.70.Ce

\section{Introduction}

Many thermodynamic quantities of solids show some peculiar features at high-temperature just before melting. Usually, this behavior is attributed to effects from both vibrational anharmonicity and thermal defects (vacancies) occurring in rare gas crystals (RGC's) in this temperature range in an appreciable concentration [1]. A phenomenological approach, taking into account anharmonic contributions to the crystal free energy [2], gives good agreement between the observed and calculated values for many thermodynamic properties of the rare gas crystals [2] and some fcc metals $(\mathrm{Cu}, \mathrm{Ag}, \mathrm{Au})$ [3]. However, the role of the vacancies and distinctive features of anharmonic contributions to the thermodynamics of these substances are not yet well understood. These problems are not easily handled in the framework of self-consistent phonon (SCP) theory [4-6], widely used for numerical investigation of crystal thermodynamics. In the present study we evaluate therefore thermodynamic characteristics of both perfect RGC's and RGC's with vacancies within the framework of a statistical theory for crystals [7], whereby a crystal is represented as an ordered ensemble of particles, whose spatial distributions are described by a binary distribution function. Recently this method has been successfully applied for the investigation of the equations of state and thermodynamic properties of rare-gas solids under pressure [8]. In Sec. 2 the main results of this statistical theory [7] are outlined and the internal energy, the heat capacities, the bulk modulus, the Grüneisen parameter and the thermal expansion are calculated for perfect crystals of Ar, Kr, Xe with contributions from anharmonicity. In Sec. 3 the vacancy contribution to the thermodynamic behavior of the RGC is added.

\section{Statistical theory of simple perfect crystals}

An effective nearest neighbor interaction energy for atoms located at the sites $R_{i}$ and $R_{j}$ in a crystal is reasonably approximated by the Morse potential

$u\left(r_{i j}\right)=A\left[\exp \left(-2 \alpha\left(r_{i j}-R_{0}\right)\right)-2 \exp \left(-\alpha\left(r_{i j}-R_{0}\right)\right)\right]$,

where $r_{i j}=\left|\mathbf{R}_{i}-\mathbf{R}_{j}+\mathbf{q}_{i}-\mathbf{q}_{j}\right|$ is the distance between atoms and $A, \alpha, R_{0}$ are the potential parameters. The values for these parameters listed in Table were determined previously [7] in such a way that the internal energy, the lattice parameter and the bulk modulus of the RGS at zero temperature and zero pressure fitted the observed values within the framework of the statistical model [7]. The determination 
of the total free energy of the crystals starts from the Gibbs-Bogoliubov functional, which includes in the present case a term for the cubic anharmonicity of the atomic vibrations $\Delta F_{3}(\tau, c)$ determined in second order perturbation theory [9]:

$$
F_{T}=F_{0}+<U-U_{0}>+\Delta F_{3}(\tau, c)
$$

whereby

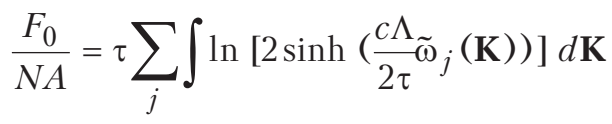

and

$$
\left.\frac{U_{0}}{N A}=\frac{c \Lambda}{4} \sum_{j} \int \tilde{\omega}_{j}(\mathbf{K}) \operatorname{coth}\left[\frac{c \Lambda}{2 \tau} \tilde{\omega}_{j}(\mathbf{K})\right)\right] d \mathbf{K}
$$

are the free energy and the average potential energy of a harmonic crystal, respectively. The integration in (3) and (4) runs over the unit cell volume of the reciprocal lattice and $K$ represents reduced components of the wave vector, varying from 0 to 1 [7].

Table

Morse potential parameters for RGC's [7,8]

\begin{tabular}{c|c|c|c|c}
\hline \hline & $\alpha, \AA^{-1}$ & $A, \mathrm{~K}$ & $R_{0}, \AA$ & $\Lambda$ \\
\hline \hline $\mathrm{Ne}$ & 2.05 & 52.98 & 3.04 & 0.4357 \\
$\mathrm{Ar}$ & 1.62 & 170.76 & 3.71 & 0.1360 \\
$\mathrm{Kr}$ & 1.52 & 249.08 & 3.97 & 0.0767 \\
$\mathrm{Xe}$ & 1.38 & 332.04 & 4.32 & 0.0457 \\
\hline \hline
\end{tabular}

The average potential energy of the interatomic interaction is calculated by the use of a binary distribution functions for the atomic displacements [7] and is given within the present approximation by

$<u>=\frac{<U>}{A N}=\exp \left(-2 b+\frac{q(\zeta)}{\gamma^{*}}\right)-2 \exp \left(-b+\frac{q(\zeta)}{4 \gamma^{*}}\right)$,

whereby

$$
\gamma^{*}(\tau)=\frac{c}{\Lambda} \sum_{j} \int \tilde{\omega}_{j}(\mathbf{K}) \tanh \left[\frac{c \Lambda}{2 \tau} \tilde{\omega}_{j}(\mathbf{K})\right] e_{i j}(\mathbf{K})^{2} d \mathbf{K}
$$

is a parameter representing both thermal and quantum broadening of the atomic motion around the lattice sites.

$$
\begin{aligned}
& \zeta(\tau)=-\frac{c}{\Lambda \gamma^{*}} \times \\
& \times \sum_{j} \int \tilde{\omega}_{j}(\mathbf{K}) \tanh \left[\frac{c \Lambda}{2 \tau} \tilde{\omega}_{j}(\mathbf{K})\right] e_{i j}(\mathbf{K})^{2} \cos \left(2 \pi K_{x} R\right) d \mathbf{K},
\end{aligned}
$$

is a parameter taking into account correlations between displacements of neighboring atoms and $q(\zeta)$ is a correlation factor, which represents the contribution of the interatomic correlation to the energy of the interatomic interactions. One may notice [7] that $q(\zeta)$ changes only slowly with temperature from $q_{0} \approx 1.87$ at $T=0 \mathrm{~K}$, to $q \approx 2$ at high temperature, where it coincides with the value for completely uncorrelated atomic states. $\tilde{\omega}_{j}(\mathbf{K})$ are the reduced frequencies determined by the dynamical matrix of the harmonic crystal, i.e. they depend on the lattice structure only, and related to the real phonon frequency $\omega_{j}(\mathbf{K})$ by

$$
\omega_{j}(\mathbf{K})=c \frac{A \Lambda}{\hbar} \tilde{\omega}_{j}(\mathbf{K}) .
$$

In terms of these variables, the crystal free energy (2) depends only on two parameters:

I) the reduced temperature $\tau=T / A$ and

II) the de Boer parameter

$$
\Lambda=\frac{\hbar \alpha}{\sqrt{M A}}
$$

which represents the strength of quantum effects ( $M$ being atomic mass). The normalized lattice expansion

$$
b(\tau)=\alpha\left(R(\tau)-R_{0}\right),
$$

depends on the parameters $\alpha$ and $R_{0}$ from the interatomic potential and on the temperature dependent

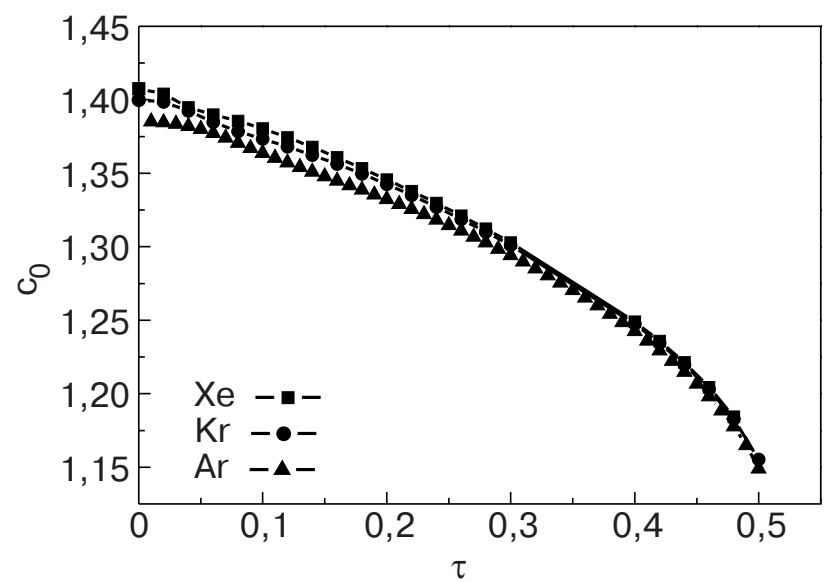

Fig. 1. Normalized temperature dependence for the quasi-elastic bond parameters of $\mathrm{Ar}, \mathrm{Kr}$ and $\mathrm{Xe}$ at ambient pressure. 
nearest neighbor distance $R$. The dimensionless quasi-elastic bond parameter

$$
c=\sqrt{\frac{\beta_{1}}{\alpha^{2} A}},
$$
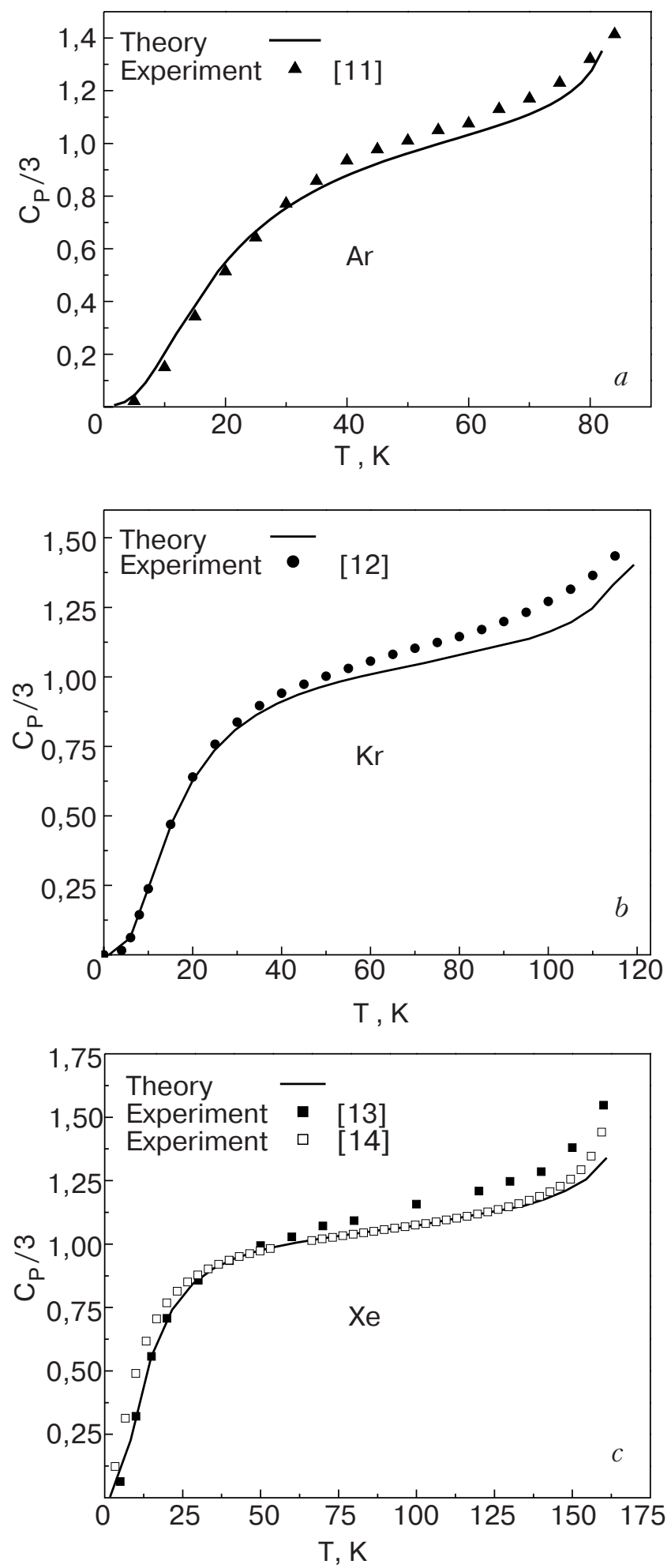

Fig. 2. Temperature dependence of the (normalized) isobaric heat capacity $C_{P}$ for $\mathrm{Ar}, \mathrm{Kr}$, and Xe at ambient pressure and experimental data points from the literature. includes an effective force constant $\beta_{1}$ for nearest neighbor atoms, and minimization of the crystal free energy (2) results in the temperature dependent equilibrium values for $b$ and $c$ denoted $b_{0}$ and $c_{0}$. This variational approach is computationally simpler than the SCP approach and gives a number of results, which are not easily obtained within the SCP model. Equations (3)-(8) allow to calculate equilibrium values for the crystal free energy (2) and for $b_{0}$ and $c_{0}$ at any temperature. Results calculated for the RGS by this procedure are illustrated in Figs. 1-6.

In Fig. 1 shows the effect of the reduced temperature $\tau$ on the quasi-elastic bond parameter $c_{0}$ for $\mathrm{Ar}$, $\mathrm{Kr}$, and Xe at zero pressure. The differences in $c_{0}$ for different crystals at low temperature are primarily caused by differences in the de Boer parameters for these elements. At a critical temperature $\tau_{c}$ close to the melting temperature, the minimum of the crystal free energy with respect to the quasi-elastic bond parameter $c$ disappears due to an increase in the anharmonicity of the atomic vibrations. These temperatures are illustrated in the Figs. 2-6 as upper ends of the calculated curves for the temperature dependencies for the interatomic distance $R(T)$, the heat capacities $C_{P}$ and $C_{V}$, the bulk modulus $K_{T}$, and the Grüneisen parameters $\gamma_{G}$ for $\mathrm{Ar}, \mathrm{Kr}$, and Xe at zero pressure, respectively. One may notice that the temperature dependence of the effective quasi-elastic bond parameter increases rapidly as the temperature approaches the instability point $\tau_{c}$. This manifests itself also in a strong non-linear increase of $C_{P}$, as well as in the thermal expansion coefficient $\alpha_{V}$ and the Grüneisen parameter $\gamma_{G}$ near the melting point (Figs. 2, 4, and 6).

In the limits of low $\left(\tau<<c_{0} \Lambda\right)$ and high $\left(\tau>>c_{0} \Lambda\right)$ temperature simple analytical expressions for (2)-(7) can be obtained [7]. For a better understanding of the peculiarities in the high temperature behavior of the RGC's and the related solid state instability, let us

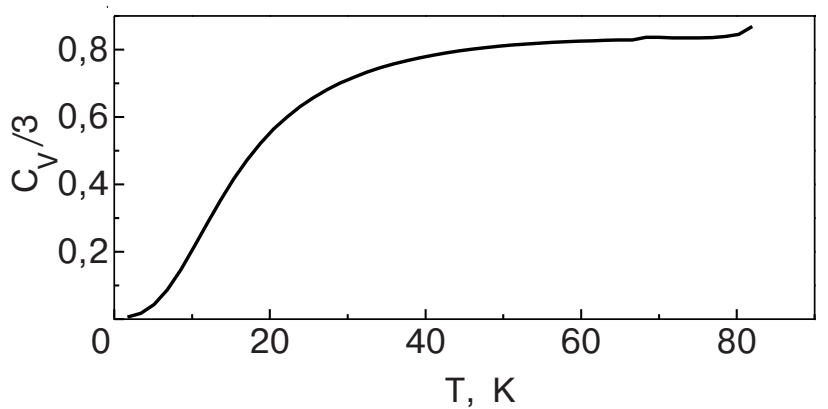

Fig. 3. Calculated (normalized) isochoric heat capacity for Ar at ambient pressure. 

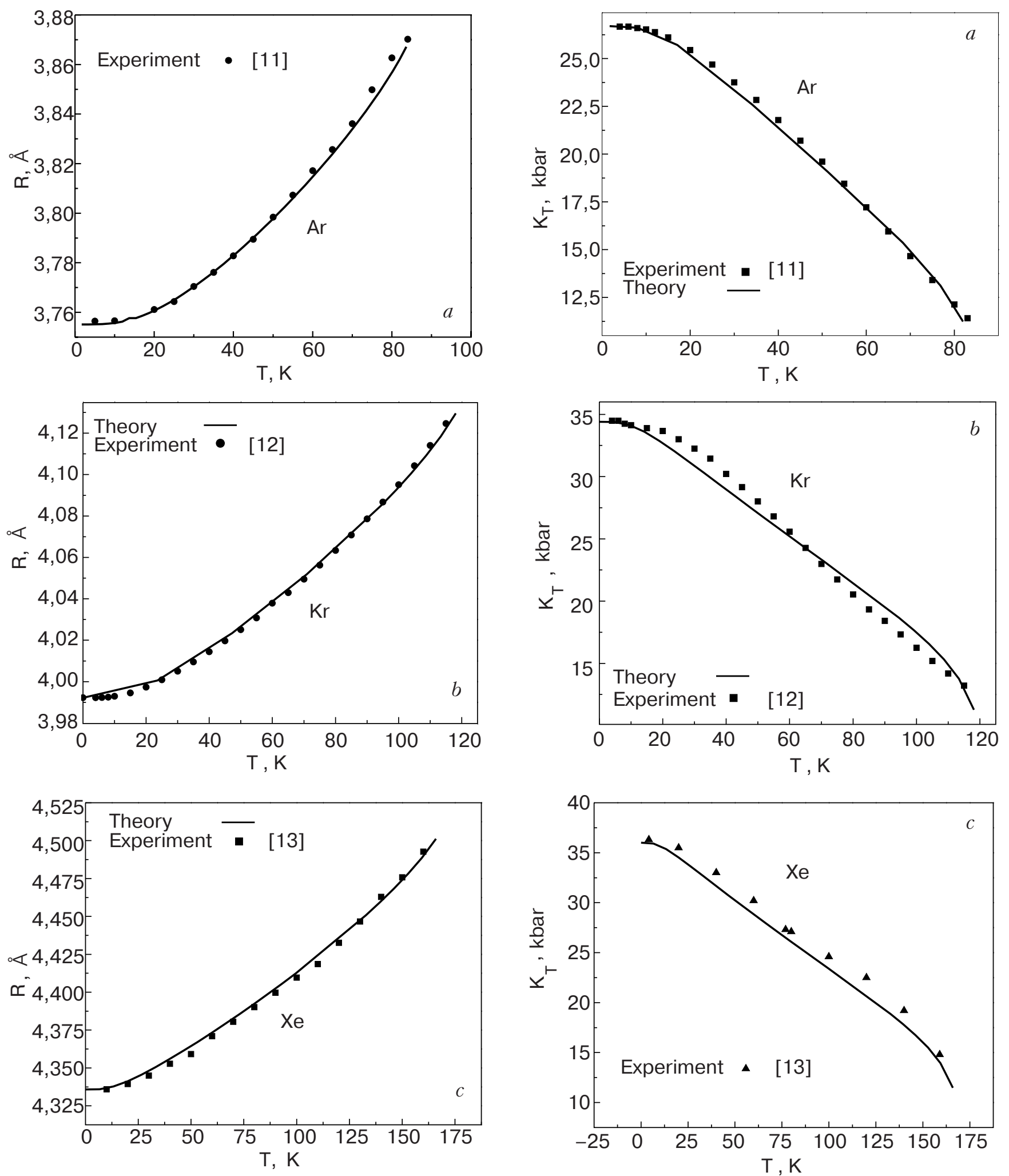

Fig. 4. Calculated temperature dependence of the nearest neighbor distance in solid Ar and Xe at ambient pressure, solid $\mathrm{Kr}$ at zero pressure and experimental data points from the literature.

Fig. 5. Calculated temperature dependence of the isothermal bulk modulus for $\operatorname{Ar}(a), \operatorname{Kr}(b)$, and Xe at ambient pressure $(c)$ and experimental data points from the literature. 
consider the classical limit $(\Lambda \rightarrow 0)$ in the expression for the free energy (2):

$$
\begin{aligned}
& \frac{F_{T}}{A N}=\frac{\tau}{3}+3 \tau \log \left[\frac{c \Lambda}{\tau}\right]+ \\
& +6\left[\exp \left(-2 b+\frac{\tau}{c^{2}}\right)-2 \exp \left(-b+\frac{\tau}{4 c^{2}}\right)\right]- \\
& -\frac{a_{3}}{c^{6}}\left[\exp \left(-2 b+\frac{\tau}{c^{2}}\right)-\frac{1}{4} \exp \left(-b+\frac{\tau}{4 c^{2}}\right)\right]^{2} \tau^{2} .
\end{aligned}
$$

The last term in (12) takes into account the contribution due to the cubic anharmonicity in the atomic vibrations at high temperature $\left(\tau \gg>c_{0} \Lambda\right)$, whereby $a_{3} \approx 1.5$ for crystals with an fcc lattice.

Minimisation of (12) with respect to $c$ and $b$ gives the equilibrium values $b_{0}$ and $c_{0}$ :

$$
\begin{gathered}
c_{0} \approx\left(1+\sqrt{1-\frac{\tau}{\tau_{c}}}\right)^{\frac{1}{2}}, \\
b_{0} \approx \frac{3 \tau}{4 c_{0}^{2}} .
\end{gathered}
$$

The value of the critical temperature $\tau_{c}$ is determined by cubic anharmonicity:

$$
\tau_{c} \approx \frac{1}{\left(1+9 a_{3} / 16\right)} .
$$

The internal energy and the isobaric heat capacity of the crystal at zero pressure and high temperature $\left(\tau \gg>c_{0} \Lambda\right)$ are given (in normalised form) by

$$
\begin{gathered}
E \approx-6+3 \tau+\frac{3 \tau^{2}}{4 c_{0}^{4}}, \\
\frac{C_{P}}{3} \approx 1+\frac{\tau}{2 c_{0}^{4}}+\frac{\tau^{2}}{4 \tau_{c} c_{0}^{6} \sqrt{1-\tau / \tau_{c}}} .
\end{gathered}
$$

For the bulk modulus $K_{T}$ and the thermal volume expansion coefficient $\alpha_{V}$ :

at $\tau \gg c_{0} \Lambda$ one obtains

$$
\begin{gathered}
K_{T} \approx \frac{4 \sqrt{2}}{3} \frac{A \alpha^{3} \exp \left(-\frac{\tau}{2 c_{0}^{2}}\right)}{\left(\alpha R_{0}+b_{0}\right)} \times \\
\times\left[\begin{array}{c}
9 \exp \left(-\frac{\tau}{2 c_{0}^{2}}\right) \tau \\
\left.1-\frac{\tau}{2 c_{0}^{4}\left[1+\frac{7}{2} \exp \left(-\frac{\tau}{2 c_{0}^{2}}\right) \frac{\tau}{c_{0}^{4}}\right]}\right]
\end{array}\right.
\end{gathered}
$$

and

$$
\alpha_{V} \approx \frac{9}{4 c_{0}^{2} A\left(\alpha R_{0}+b_{0}\right)}\left[1+\frac{\tau}{2 c_{0}^{2} \tau_{c} \sqrt{1-\frac{\tau}{\tau_{c}}}}\right]
$$

Minor contributions from terms $\sim a_{3} \tau^{2}$ are neglected in the Eqs. (17)-(19), which represent obviously very well the qualitative behavior of the RGC's at high temperature. The relations (13)-(19) show that the main internal parameter, governing the thermodynamic behavior of a simple crystal at high temperature, is the effective quasi-elastic bond parameter $c_{0}(\tau)$, which depends parametrically also on the reduced critical temperature $\tau_{c}$. From the linear relation between the sound velocities $u_{j k}$ and the quasi-elastic bond parameter

$$
c_{0}(\tau)=\left(\frac{m}{A \alpha^{2}}\right)^{\frac{1}{2}} \frac{u_{j \mathbf{k}}}{\kappa_{j \mathbf{k}} R(\tau)},
$$

with the coefficients $\kappa_{j \mathbf{k}}$ depending on the polarization $j$ and on the $\mathbf{k}$-vectors of the sound waves, one obtains for the commonly used (acoustic) Debye temperature

$$
\Theta_{D}=\left(6 \sqrt{2} \pi^{2}\right)^{\frac{1}{3}}<\kappa_{j \mathbf{k}}>>A \Lambda c_{0}(\tau)
$$

whereby $\left\langle\kappa_{j \mathbf{k}}>\approx 0.677\right.$ for the fcc lattice. This direct relation between the (acoustic) Debye temperature and the quasi-elastic bond parameter indicates, that the temperature and volume dependence of $c_{0}(\tau)$ can be use in the determination of all the thermodynamic properties of these crystals in the same way as the Debye temperature in the commonly applied Debye model, however in the present case, the tem-

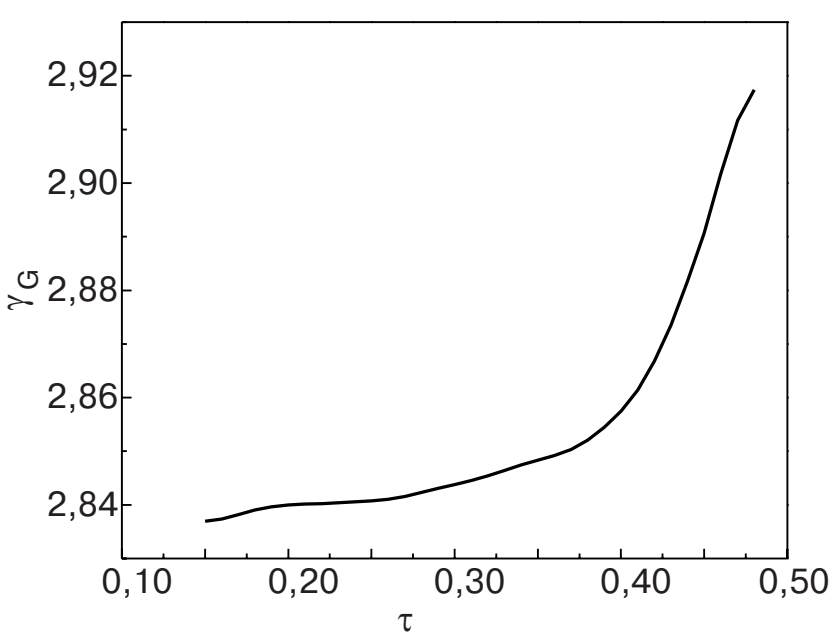

Fig. 6. Calculated temperature dependence of the thermal Grüneisen parameter for Ar at ambient pressure. 
perature dependence of $c_{0}(\tau)$ includes also anharmonic contributions, which are not included in the commonly used quasiharmonic Debye approach.

\section{Contributions of vacancies to the thermodynamic properties}

Vacancy contributions to thermodynamic properties of crystals are determined primarily by their equilibrium concentrations

$$
n_{v}=\exp \left(-g_{v} / T\right)
$$

where $g_{v}=h_{v}-T s_{v}$ is the excess Gibbs energy associated with the vacancies and $h_{v}=\varepsilon_{v}+P v_{v}$ and $s_{v}$ are the corresponding enthalpy and entropy, respectively.

The broken bonds result in local deformations around the vacant sites and in changes of the quasi-elastic bond parameters for the surrounding atoms. The total contribution to the Gibbs energy of vacancy formation is then represented by [14]

$$
g_{v}=-2.33 A \tau+3.77 A .
$$

The vacancy contribution to the isobaric heat capacity (at zero pressure) of a simple crystal is given within this approximation by

$$
C_{\mathrm{vac}}=\left[\frac{h_{\mathrm{vac}}}{T}\right]^{2} \exp \left(-g_{v} / T\right) .
$$

As can be seen from (24), the largest contribution of the vacancies to the heat capacity of the crystal should be seen at the highest temperatures. In the present cases $C_{\mathrm{vac}} / 3<<1$, i.e. the contribution of vacancies to the total heat capacity of the crystal remains small compared with the lattice contributions even at the highest temperatures.

The change of the crystal volume under pressure depends on both the lattice compression and the decrease in the number of vacancies:

$$
\frac{d V}{d P}=-\frac{1}{K_{T}^{0}} V+v_{v} \frac{d n_{\mathrm{vac}}}{d P}
$$

where $K_{T}^{0}$ is the bulk modulus of a perfect crystal. The pressure dependence in the number of vacancies is determined by the enthalpy $h_{v}=\varepsilon_{v}+P v_{v}$ of the vacancy formation. In this case the effective bulk modulus of the crystal with defects can be written as

$$
K_{T}=\frac{K_{T}^{0}}{1+\frac{\alpha^{3} v_{v}}{\tau} n_{\mathrm{vac}} K_{T}^{0}} .
$$

An estimate of the contributions in (26) shows that the denominator is close to one at any temperature, i.e. contributions from vacancies to the bulk modulus of the RGC's are very small and can be safely neglected.

In the literature [15] similar conclusions can be found with respect to the thermodynamic contribution of the vacancies in the case of copper.

This research was supported in parts by an award from the Deutscher Akademischer Austauschdienst (DAAD) to A.I.K.

1. D.L. Losee and R.O. Simmons, Phys. Rev. 172, 934 (1968).

2. W.B. Holzapfel, M. Hartwing, and G. Reiß, J. Low Temp. Phys. 122, 401 (2001).

3. W.B. Holzapfel, High Press. Res. 16, 81 (1998).

4. N. Boccara and G. Sarma, Physics 1, 219 (1965) (Long Island City, New York).

5. T.R. Koehler, Phys. Rev. Lett. 17, 89 (1966).

6. H. Horner, Z. Phys. 205, 72 (1967).

7. A.I. Karasevskii and V.V. Lubashenko, Phys. Rev. B66, 054302 (2002).

8. A.I. Karasevskii and W.B. Holzapfel, Phys. Rev. B67, 224301 (2003).

9. V.V. Goldman, G.K. Horton, and M.L. Klein, Phys. Rev. Lett. 21, 1527 (1968).

10. O.G. Peterson, D.N. Batchelder, and R.O. Simmons, Phys. Rev. 150, 703 (1966).

11. D.L. Losee and R.O. Simmons, Phys. Rev. 172, 944 (1968).

12. P. Korpiun and E. Luscher, Rare Gas Solids, Academic Press, London, vol. 2 (1977), p. 729.

13. P. Korpiun and H.J. Coufal, Phys. Status Solidi A10, 187 (1971).

14. S.Sh. Soulayman, J.V. Zanchetta, and J.C. Giuntini, J. Chem. Phys. 102, 7641 (1995).

15. D. Acoccella, G.K. Horton, and E.R. Cowley, Phys. Rev. B61, 8753 (2000). 